\title{
George C. Sutton, Kanu Chatterjee: Heart failure: current clinical understanding
}

\author{
REMEDICA, London and Chicago, www.remedicabooks.com, 58 pages
}

\author{
Sidney Goldstein
}

Published online: 14 November 2009

(C) Springer Science+Business Media, LLC 2009

This beautifully illustrated book is ideal for the new physician or health care associate interested in becoming initiated in the current state of the knowledge of heart failure diagnosis and therapy. The research and drug development that has occurred in the last half century is clearly described in this monograph by two of the world's most outstanding cardiologists, Dr. George C. Sutton at the Imperial College London and Dr. Kanu Chatterjee at the University of California. Sections on pathology and diagnosis are clearly integrated to provide a clear relationship between physical findings and pathophysiology. Pathologic specimens are also integrated into the latest advances in diagnostic technology. In addition, an incisive review of the major knowledge about the relationship between disease mechanisms, particularly the renin-angiotensin system and disease progression, is also developed in the text. The ever-changing state of drug and surgical therapy is well covered and provides physiologic guidelines to facilitate the choice of the optimal therapy for different heart failure etiologies. In addition, an excellent review of the epidemiology of the causes of heart failure is presented. All in all this is a small but very inclusive monograph that opens the door to the understanding of the complexities of diagnosis and therapy of heart failure in contemporary medicine. 